\title{
PERFORMANCE IMPROVEMENT OF NON- REgENERATIVE COOPERATIVE RELAY NETWORKS With OPTIMAl PoWer Allocation AND ADAPTIVE M-QAM/M-PSK MODULATION OVER GENERALIZED FADING CHANNELS
}

\author{
Bhuvan Modi, A. Annamalai, O. Olabiyi and O.Odejide \\ Center of Excellence for Communication Systems Technology Research \\ Department of Electrical and Computer Engineering, Prairie View A \& M University, \\ TX 77446 United States of America
}

\begin{abstract}
This paper analyzes the performance of Channel Side Information (CSI)-assisted cooperative amplify-andforward (CAF) relay networks that employ both the node placement (i.e., relay position) based optimal power allocation policy among collaborating nodes and adaptive M-ary quadrature amplitude modulation $(M-Q A M) / M$-ary phase shift keying (M-PSK) techniques in generalized wireless fading environments. In particular, we advocate a simple yet unified numerical approach based on the marginal moment generating function $(M G F)$ of the total received Signal to Noise Ratio (SNR) to derive analytical expressions for the average bit error rate (ABER), mean achievable spectral efficiency, and outage probability performance metrics. The proposed analytical framework is sufficiently general and flexible to characterize the performance of adaptive-link CAF relay networks over a wide range of fading distributions (i.e., not restricted to Rayleigh fading or independent identically distributed (i.i.d) Nakagami-m fading ) with independent but non-identically distributed (i.n.d) fading statistics across the spatially distributed diversity paths. Additionally, we further simplify the computational complexity, by employing the use of an "approximate MGF expression" to compute the system performance metrics over the generalized fading channel. Employing the above novel approach based on "approximate MGF" in conjunction derived analytical frameworks allows us to simplify the computation complexity of achievable spectral efficiency as well as ABER of CAF relay system in the generalized fading environments by simply replacing appropriate single channel MGF which is readily available.
\end{abstract}

\section{KEYWORDS}

Cooperative diversity, adaptive modulation, optimum power allocation, relay node placement

\section{Motivation}

The increasing demand for wireless communication system in recent years has necessitated a need to improve the performance, reliability and data rate of wireless channels. To address these needs, several attempts have been made on each of these improvement areas. The broadcast nature of wireless transmissions has enabled a new communication paradigm known as "cooperative communication" where a source node communicates with a destination node with the help of one or more relay nodes to harness a new form of spatial diversity and combat the effect of multipath fading. Cooperative communication serves as an alternative to conventional space diversity technique (i.e., Multiple Input Multiple Output (MIMO) technique), as the size of communication nodes (i.e., mobile handheld devices or wireless sensors) may not accommodate multiple transmit and/or receive antennas. Cooperative diversity also referred to as a "distributed MIMO" provides additional reliability to the wireless system. 
Link adaptation is yet another powerful wireless communication strategy for improving the spectral utilization efficiency; wherein the power level, signal constellation size and/or coding rate are "matched" with the prevailing channel conditions based on the acquired channel side information on feedback channel.

Integrating these two powerful communication techniques (i.e., cooperative communications and link adaptation) could benefit the emerging IEEE 802.16 wireless networks (i.e., the current IEEE 802.16e systems employ adaptive modulation, while the emerging IEEE 802.16j standard specifies the use of cooperative diversity in its multi-hop relay architecture).

In this paper, our main focus on the link-adaptive amplify-and-forward relaying strategy because it does not require "sophisticated" transceivers at the relays (although our framework is also applicable for digital relaying, once the moment generating function is found or available). While this protocol can achieve full diversity using a virtual antenna array, there is a loss of spectral efficiency due to its inherent half-duplex operation. But this penalty could be "recovered" by combining the cooperative diversity with a link adaptation mechanism wherein the power level, signal constellation size, coding rate or other transmission parameters are adapted autonomously in response to fluctuations in the channel conditions.

There has been widespread research on performance analyses of both non-adaptive cooperative diversity systems and adaptive transmission techniques for classical (non-cooperative) wireless networks; however the art of adaptive link layer in cooperative wireless networks is still in its infancy especially when optimized in a cross-layer design paradigm. For instance majority of the literature on the cooperative relay diversity are limited to both fixed signaling rate and/or constant transmit power for all communication nodes. A review of prior research on cooperative diversity with adaptive transmission is discussed next. Authors in [1]-[2] studied the problem of the optimal power allocation in wireless relay networks, but they did not consider source rateadaptation. Adaptive transmission for a two-hop (regenerative and non-regenerative) relay network (but without the direct source-destination link) in the Rayleigh fading channel was investigated in [3]. In [4], authors have studied the effect of power allocation and relay location on system performance for decode and forward dual-hop system over a Nakagami-m fading environment, but without a direct source to destination link, and more importantly without considering adaptive modulation scheme. Whereas authors in [5] studied the power assignment strategy by considering fixed relay position for incremental selective decode and forward cooperative relay networks over the Rice fading channel in terms of only symbol error rate analysis, but without link adaptation. Authors in [6] studied joint relay selection and adaptive modulation coding in amplify and forward relay network with Rayleigh fading channel. The ABER performance of a cooperative relay network with BPSK modulation in a Nakagami-m fading channel was investigated in [7], however the authors did not consider higher constellation size or adaptive modulation schemes. The performance of a CAF relay network with constant power M-QAM adaptive rate transmission, when the ABER in Rayleigh fading is constrained to be below a specified target bit error rate (BER) is examined in [8] for optimum mode switching thresholds. In [9], the performance of discrete-rate adaptive M-QAM for a single incremental relay in a Nakagami-m environment was examined. While authors in [10] investigate the performance of a cooperative decode-and-forward relay network with five-modes adaptive $\mathrm{M}$ QAM transmission in an i.i.d Nakagami-m wireless fading environment. It is interesting to note that, authors in [11] derived bounds for the outage probability, achievable spectral efficiency, and error rate performance of a link-adaptive amplify-and-forward cooperative diversity system with limited CSI, in which the rate at the source node is adapted according to channel conditions (i.e., only feedback of the effective SNR at the destination node is required to be available at the source node), while the relays simply amplify and forward the signals. For the analysis, they considered the MGF based approach to compute the above performance metrics using adaptive M-QAM 
technique in CAF relay networks. However, their framework is limited to only the Rayleigh fading channel, and more importantly, the power allocation and the node placement strategies were not considered, which can have a significant impact on overall system performance.

Motivated by these observations, our main contributions in this paper are summarized as follows:

\section{i)Development of flexible generalized analytical framework}

We develop a unified generalized analytical framework based on the marginal MGF and/or the Cumulative Distribution Function (CDF) method for evaluating the ABER as shown in eq (13), mean spectral utilization efficiency in (12) and outage probability performance metrics of the CAF relay networks with both link adaptive-techniques (i.e., adaptive discrete rate (ADR) MQAM and M-PSK) for generalized fading environments (i.e., as long as the MGF of total end-toend SNR may be readily available for desired fading channel). In this paper, we significantly extend the analysis of CAF relay networks with constant power M-QAM adaptive rate transmission in [11] to a more general stochastic fading environment (i.e, [11] presented the results for only Rayleigh fading channel). Specifically, we analyze the performance of CAF relay networks with both ADR M-QAM and M-PSK modulation for improving the average link spectral efficiency, while satisfying a prescribed instantaneous BER performance. More importantly, in contrast to [9], [10] and [11], we come up with a new analytical framework (i.e., generalized to wide range of fading environment, once the MGF of end-to-end SNR of a particular fading environment is readily available) based on the marginal MGF for evaluating the ABER, mean spectral utilization efficiency and outage probability performance metrics. Extending the probability density function (PDF) method employed in [9], [10]-[11] to a generalized fading channel with i.n.d fading statistics appears intractable or very cumbersome. It is also interesting to note that even for the specific case of the Rayleigh fading, our mean spectral efficiency performance curves computed (using the MGF shown in (16)) for the ADR MQAM/M-PSK scheme are considerably more closer than the closed-form upper and lower bounds derived in [11].

\section{ii)Application of our generalized analytical framework to present special case of Rice fading channel (Please refer figure 5)}

We utilized our proposed flexible/compatible generalized analytical frameworks developed in (12) and (13) to present the special case of Rice fading channel. For that we employ the readily available closed form MGF of upper and lower bounds of the harmonic mean SNR of two-hop CAF relay networks over the Rice fading channel derived from [12, eq. (6)] and applied to our developed equations (12) and (13) to compute spectral efficiency and ABER respectively. To the best of our knowledge the analysis of CAF relay network in terms of ABER and mean spectral efficiency in the Rice fading environment has never been considered in earlier publications. The reason being that the evaluation of the above performance metrics require tractable expression for the probability density function (PDF), cumulative distribution function (CDF) and/or MGF of end-to-end SNR which has been difficult to obtain for the Rice fading channel. It is worth to mention that the exact MGF of harmonic mean SNR for the relayed path presented in [13, Eq. (5) and Table 1] for the Rice fading channel is not tractable as it involves the integral of the product of two infinite series (with Bessel function arguments). Therefore, we first adopt a tractable and closed form MGF of upper and lower bounds of harmonic mean SNR over the Rice fading environments (similar to [14] for Rayleigh fading). Moreover, for the computation of above performance metrics we need to compute the inverse Laplace transform of the closed form MGF expression for upper and lower bound derived in [12, eq. (6)].This inversion requires the evaluation of the Marcum-Q function (i.e., See (17)) with a complex argument, which is not available in the built-in function of the most computing software. So, we overcome this limitation 
by incorporating a new routine to compute this using a rapidly convergent canonical series representation of the Marcum Q-function developed in [12, eq., (10)] to efficiently evaluate the mean spectral efficiency, outage probability and ABER performance parameters in case of Rice fading channel.

\section{iii)Derived closed form expression for the marginal MGF of end-to-end SNR of CAF relay system over an i.n.d Nakagami-m fading channel (please refer section 2.1)}

Motivated by the need for closed form solution, we derived closed form expression for the marginal MGF of end-to-end SNR of CAF relay system over an i.n.d Nakagami-m fading channel by the use of partial fraction, and then employ the resulting expression in direct evaluation of performance metrics of CAF relay system. However, it is worth to mention that, the partial expansion decomposition is only applicable for integer $\mathrm{m}$ ( $\mathrm{m}$ is the Nakagami fading parameter) and more tedious for multiple relay system. Therefore, from computational efficiency perspective, it is considerably simpler to program and evaluate the desired ABER, mean spectral utilization efficiency and outage probability, using closed form MGF (in conjunction with FixedTalbot method [15]) for non-integer $\mathrm{m}$ and high diversity order for any fading environments (i.e., since the MGF of total received SNR may be easier to compute or readily available for CAF relay networks).

iv)Development of system model by employing approximate MGF for generalized fading channel (please refer section 2.2 and Figure 5)

Moreover, in an attempt to further simplify the computation complexity, we also employ the use of an "approximate MGF expression" from [16, Eq. (12)] to compute the system performance metrics using our generalized analytical framework developed in (12) and (13). It is interesting to note that this MGF expression, despite its simplicity, closely approximates the MGF of harmonic mean SNR and can handle generalized i.n.d. fading channels and mixed fading environments (i.e., see (7)).

v)Application of developed flexible analytical framework to relay position based optimal power allocation strategy (Please refer Figure 7 and 8)

Besides, in contrast to [8], [9], [11] and [14]; and for the completeness of the paper, we also applied our flexible framework derived in (12) and (13) to investigate the efficacy of node placement (i.e., relay position) based optimal power allocation during different transmission phases in a link-adaptive CAF relay networks (i.e., complete CSI case since the source-node also requires the knowledge of channel gains of all links) to optimize the mean spectral efficiency which can have significant impact on system performance. This shows that our developed analytical framework can also support/incorporate the analysis of relay node placement based optimal power allocation strategy without developing separate dedicated mathematical model.

The remainder of this paper is organized as follows. In Section 2, the system model is briefly discussed. Section 3 derives the performance metrics for CAF relay networks with adaptive MQAM/M-PSK modulation. Selected numerical results are presented in Section 4. Our conclusions are given in Section 5. In appendixes A and B we present numerical procedure to derive closed form marginal MGF from the readily available MGF of upper bound of end-to-end SNR in an i.n.d Nakagami-m fading channel. 


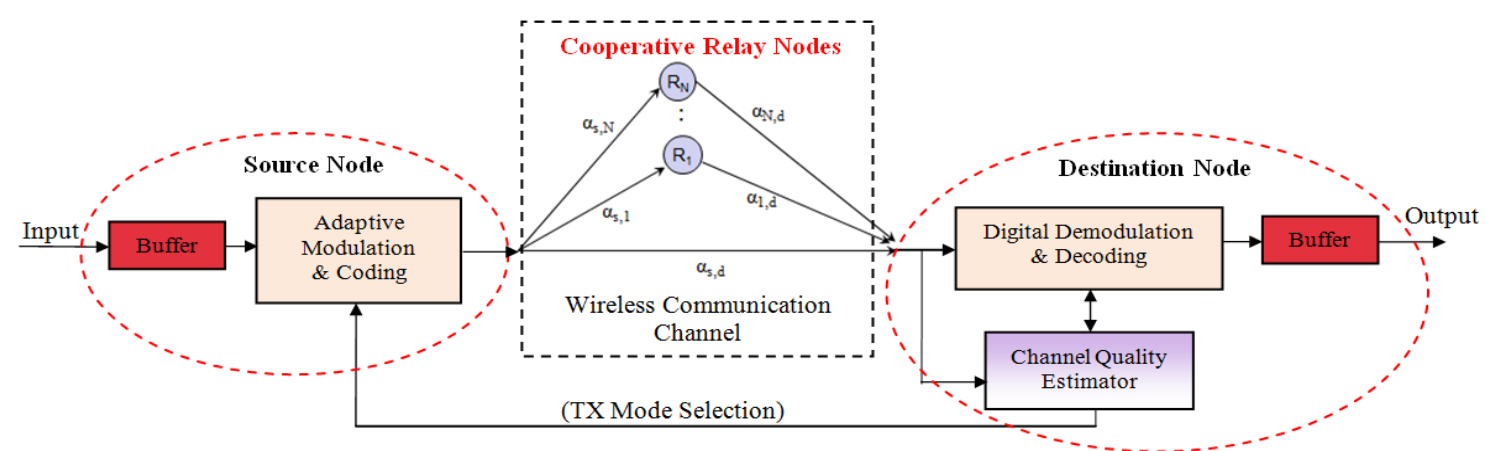

Figure 1: Link-adaptive cooperative diversity system for ensuring the connectivity and network stability needed to support varying quality-of-service requirements.

Table I: Optimized coefficients of approximate BER for M-PSK and M-QAM in AWGN channels

\begin{tabular}{|c|c|c|c|c|c|}
\hline & Mode 1 & Mode 2 & Mode 3 & Mode 4 & Mode 5 \\
\hline M-PSK & $\mathbf{2}$ & $\mathbf{4}$ & $\mathbf{8}$ & $\mathbf{1 6}$ & $\mathbf{3 2}$ \\
\hline $\boldsymbol{a}_{\boldsymbol{n}}$ & $1.6214 \mathrm{E}-1$ & $8.9403 \mathrm{E}-4$ & $1.0267 \mathrm{E}-1$ & $7.1904 \mathrm{E}-2$ & $6.1306 \mathrm{E}-2$ \\
\hline $\boldsymbol{b}_{\boldsymbol{n}}$ & $1.0645 \mathrm{E}+0$ & $2.9904 \mathrm{E}-1$ & $1.5510 \mathrm{E}-1$ & $3.9760 \mathrm{E}-2$ & $1.0160 \mathrm{E}-2$ \\
\hline $\boldsymbol{c}_{\boldsymbol{n}}$ & $1.9975 \mathrm{E}-1$ & $2.8202 \mathrm{E}-1$ & $1.6718 \mathrm{E}-1$ & $1.2993 \mathrm{E}-1$ & $9.7244 \mathrm{E}-2$ \\
\hline M-QAM & $\mathbf{2}$ & $\mathbf{4}$ & $\mathbf{1 6}$ & $\mathbf{6 4}$ & $\mathbf{2 5 6}$ \\
\hline $\boldsymbol{a}_{\boldsymbol{n}}$ & $1.6214 \mathrm{E}-1$ & $8.9403 \mathrm{E}-4$ & $1.1002 \mathrm{E}-1$ & $8.2198 \mathrm{E}-2$ & $6.2349 \mathrm{E}-2$ \\
\hline $\boldsymbol{b}_{\boldsymbol{n}}$ & $1.0645 \mathrm{E}+0$ & $2.9904 \mathrm{E}-1$ & $1.0541 \mathrm{E}-1$ & $2.5002 \mathrm{E}-2$ & $6.1622 \mathrm{E}-3$ \\
\hline $\boldsymbol{c}_{\boldsymbol{n}}$ & $1.9975 \mathrm{E}-1$ & $2.8202 \mathrm{E}-1$ & $2.1261 \mathrm{E}-1$ & $1.8771 \mathrm{E}-1$ & $1.9314 \mathrm{E}-1$ \\
\hline
\end{tabular}

\section{SYSTEM MODEL}

Link-adaptive cooperative wireless system model is shown in Figure 1. Source node $S$ communicates with a destination node $D$ via a direct-link and through $N$ amplify-and-forward relays, $R_{i}, i \in\{1,2, \ldots, N\}$, in two transmission phases. During Phase I, $S$ transmits signal $x$ to $D$ as well as to the relays $R_{i}$, where the channel fading coefficients between $S$ and $D, S$ and the $i$-th relay node $R_{i}$; and $R_{i}$ and $D$ are denoted by $\alpha_{s, d}, \alpha_{s, i}$ and $\alpha_{i, d}$ respectively. During the second phase of cooperation, each of the $N$ relays transmits the received signal after amplification via orthogonal transmissions (e.g., Time Division Multiple Access (TDMA) in a round-robin fashion and/or Frequency Division Multiple Access (FDMA)). Suppose $P_{i, d}$ is the transmit power of $i$-th relay node while transmitting its signal to the destination node and $P_{s, d}=P_{s, i}$ denotes the transmitted power at the source node. We also assume that the total transmitted power $P_{s, d}+\sum_{i=1}^{N} P_{i, d}=P_{T}$ is fixed.

Now consider that the maximum ratio combiner (MRC) is employed at a $D$ to coherently combine all the signals received during Phase I and Phase II, the total received SNR at output of the MRC detector can be shown to be [17], [18], [29]

$\gamma=\gamma_{s, d}+\sum_{i=1}^{N} \frac{\gamma_{s, i} \gamma_{i, d}}{1+\gamma_{s, i}+\gamma_{i, d}}=\gamma_{s, d}+\sum_{i=1}^{N} \gamma_{i} \leq \gamma_{s, d}+\sum_{i=1}^{N} \gamma_{i}^{(H M)}$ 
where $\gamma_{i}^{(H M)}=\frac{\gamma_{s, i} \gamma_{i, d}}{\gamma_{s, i}+\gamma_{i, d}}$ is the harmonic mean SNR, $\gamma_{i}=\frac{\gamma_{s, i} \gamma_{i, d}}{1+\gamma_{s, i}+\gamma_{i, d}}$ and $\gamma_{s, i}=\left|\alpha_{s, i}\right|^{2} P_{s, i} T_{s} / N_{0}$ and $\gamma_{i, d}=\left|\alpha_{i, d}\right|^{2} P_{i, d} T_{s} / N_{0}$ denote the instantaneous SNR of the source-relay and the relay-destination links respectively, while $T_{s}$ corresponds to the symbol duration. The bound on the right-side of (1) is obtained by recognizing that the instantaneous SNR of a two hops path can be accurately estimated to be the half harmonic mean of individual link SNRs especially at moderate and high SNR regimes [19]. Suppose $\gamma_{s, d}, \gamma_{s, i}, \gamma_{i, d}$ are i.n.d random variables, it is straight forward to show that the MGF of $\gamma$ in (1) is given by

$$
\phi_{\gamma}(s)=\phi_{\gamma_{s, d}}(s) \prod_{i=1}^{N} \phi_{\gamma_{i}}(s)
$$

where $\phi_{\gamma_{s, d}}(s)$ corresponds to the MGF of SNR of the $S$ - $D$ link while $\phi_{\gamma_{i}}(s)$ denotes the exact MGF of end-to-end SNR for a dual-hop relayed path. However, finding $\phi_{\gamma_{i}}(s)$ for a generalized fading environment can be a very daunting task, with existing results limited to only Rayleigh fading [20] and Nakagami-m [21] fading environments. Even in such cases, the final expressions are too complicated for further manipulations. For example, the exact MGF of SNR for a dual-hop CAF with i.n.d Nakagami-m fading statistics derived in [21] involves triple summation terms involving $k^{\text {th }}$ derivative of a product of Whittaker functions, which is not easily evaluated using a general computing platform, besides being restrictive to positive integer values for the fading severity index. Other "exact" formulas (i.e., half-harmonic mean bound of the exact end-to-end SNR $\gamma_{i}^{(H M)}$ ) for the MGF of SNR in a relayed path can be found in [22] (for i.n.d Rayleigh channels), [19] (for i.i.d Nakagami-m channels) and [13]. While the MGF-based approach developed in [13] is quite interesting and can be applied to a wide range of fading environments, the resulting integral expressions are often too complicated to compute and/or time-consuming (due to the need to evaluate a nested two-fold integral term with complicated arguments that might include infinite series in some cases such as Rice fading). To circumvent the aforementioned difficulties, both upper and lower bounds have been proposed and developed for $\gamma_{i}^{(H M)}$, viz., [14], [23]

$$
\gamma_{i}^{(L B)}=\frac{1}{2} \min \left(\gamma_{s, i}, \gamma_{i, d}\right) \leq \gamma_{i}^{(H M)} \leq \gamma_{i}^{(U B)}=\min \left(\gamma_{s, i}, \gamma_{i, d}\right)
$$

Hence approximate performance bounds can be developed by utilizing the bounds for the MGF of total received SNR using the inequality in [11], [24] (i.e., see (3)) viz.

$$
\phi_{\gamma_{s, d}}(s) \prod_{i=1}^{N} \phi_{\gamma_{i}}^{(U B)}(s) \leq \phi_{\gamma}(s) \leq \phi_{\gamma_{s, d}}(s) \prod_{i=1}^{N} \phi_{\gamma_{i}}^{(L B)}(s)
$$

where $\phi_{\gamma_{i}}^{(U B)}(s)$ and $\phi_{\gamma_{i}}^{(L B)}(s)=\phi_{\gamma_{i}}^{(U B)}(s / 2)$ corresponds to the MGFs of $\gamma_{i}^{(U B)}$ and $\gamma_{i}^{(L B)}$ respectively.

\subsection{Marginal MGF of End-to-End SNR of CAF Relay System over i.n.d Nakagami- m Fading Channel}

In this section we will derive closed form expression for the marginal MGF of end-to-end SNR of CAF relay system over an i.n.d Nakagami-m fading channel by the use of partial fraction (see details in Appendices A \& B), and then employ the resulting expression in direct evaluation of performance metrics of CAF relay system. 
The MGF for upper bound of $\gamma_{i}^{(U B)}$ for two-hop relayed path in a Nakagami-m channel with i.n.d fading statistics is given by [12]

$\phi_{\gamma_{i}}^{(U B)}(s)=\sum_{\substack{k \in\{(s, i),(i, d)\} \\ j \neq k}} \frac{\Gamma\left(m_{k}+m_{j}\right)}{m_{k} \Gamma\left(m_{k}\right) \Gamma\left(m_{j}\right)}\left(\frac{\Omega_{j} m_{k}}{s \Omega_{j} \Omega_{k}+\Omega_{j} m_{k}+\Omega_{k} m_{j}}\right)^{m_{k}}{ }_{2} F_{1}\left(1-m_{j}, m_{k} ; 1+m_{k} ; \frac{\left(s \Omega_{k}+m_{k}\right) \Omega_{j}}{s \Omega_{j} \Omega_{k}+\Omega_{j} m_{k}+\Omega_{k} m_{j}}\right)$

where $\Omega_{q}=E\left[\gamma_{q}\right]$ corresponds to the mean SNR of link $q, \Gamma($.$) is the Gamma function and m_{q}$ is the Nakagami-m fading index.

Utilizing (A.4) and (B.4), the closed form marginal MGF for upper bound of $\gamma$ in (2) can be written as,

$$
\begin{aligned}
& \phi_{\gamma}^{(U B)}(s, \gamma)=\sum_{\substack{\{(s, i)(i, d)\} \\
j \neq k}} \frac{\Gamma\left(m_{k}+m_{j}\right)}{\Gamma\left(m_{k}\right) \Gamma\left(m_{j}\right)} \sum_{u=0}^{m_{j}-1} \frac{(-1)^{u}}{\left(m_{k}+u\right)}\left(\begin{array}{c}
m_{j}-1 \\
u
\end{array}\right) \times \sum_{r=0}^{u}\left(\begin{array}{l}
u \\
r
\end{array}\right)(-1)^{-r}\left(1+\frac{\Omega_{k} m_{j}}{\Omega_{j} m_{k}}\right)^{-m_{k}}\left(1+\frac{\Omega_{j} m_{k}}{\Omega_{k} m_{j}}\right)^{-r} \\
& \times\left[\sum_{p=1}^{m_{s}} \frac{\alpha_{p} G\left(p,\left(s+\frac{m_{s}}{\Omega_{s}}\right) \gamma\right)}{\Gamma(p)\left(1+\frac{\Omega_{s}}{m_{s}} s\right)^{p}}+\sum_{q=1}^{m_{k}+r} \frac{\alpha_{q} G\left(q,\left(s+\frac{\Omega_{j} m_{k}+\Omega_{k} m_{j}}{\Omega_{j} \Omega_{k}}\right) \gamma\right]}{\Gamma(q)\left(1+\frac{\Omega_{j} \Omega_{k}}{\Omega_{j} m_{k}+\Omega_{k} m_{j}} s\right)^{q}}\right]
\end{aligned}
$$

where $\alpha_{p}$ and $\alpha_{q}$ are defined in (A.4) and $G(v, x)=\int_{0}^{x} t^{p-1} e^{-t} d t$ is the lower incomplete gamma function.

\subsection{Approximate MGF of SNR in Mixed Fading Channels}

Along with the bounding techniques, for further simplicity, we also employ an "accurate approximate MGF" of $\gamma_{i}$ for two-hop relayed path in (2) which is given by [16, Eq. (12)],

$$
\phi_{\gamma_{i}}^{(A)}(s) \approx \phi_{r_{s, i}}(s)+\phi_{\gamma_{, d}}(s)-\phi_{r_{s, i}}(s) \phi_{\gamma_{i, d}}(s)
$$

where $\phi_{\gamma_{a, b}}(s)$ is the MGF of a single channel for link $a$ to $b$. Above expression allows us to simplify the computation complexity of the performance metrics in the CAF relay system under generalized fading environments, as it does not give any problem with the inverse Fourier/Laplace transformation. It is also interesting to note that, the MGF expression in (7) is simple and can readily handle complex and mixed fading channel models. In fact, it is sufficiently general over a wide range of fading environments and can be easily evaluated by substituting appropriate single channel MGF, which is readily available for various fading environments in [25, Table 2.2]. In this paper we also employ above flexible approximate MGF expression in (7) to our developed flexible/compatible equations (12) and (13) to easily analyse the spectral efficiency and ABER for wide range of fading environments.

\subsection{Cumulative Distribution function (CDF) of the CAF Relay Networks.}

The knowledge of the CDF of total effective SNR $\gamma$ of the CAF relay networks is required for the evaluation of various performance measures. Since the analytical CDF expression for the CAF relay networks is difficult to obtain, the alternative is to compute the CDF from the MGF 
expression in (2). One of the most efficient frequency inversion method is the Abate's FixedTalbot method (i.e., multi-precision Laplace transform inversion) [15], viz.,

$$
F_{X}(x) \cong \frac{1}{2 Z} \phi_{X}(r) e^{r x}+\frac{r}{Z} \sum_{k=1}^{Z-1} \operatorname{Re}\left\{\frac{1+j \sigma\left(\theta_{k}\right)}{s\left(\theta_{k}\right)} e^{x s\left(\theta_{k}\right)} \phi_{X}\left(s\left(\theta_{k}\right)\right)\right\}
$$

where $\quad r=2 Z /(5 x), \theta_{k}=k \pi / Z, \sigma\left(\theta_{k}\right)=\theta_{k}+\left(\theta_{k} \cot \left(\theta_{k}\right)-1\right) \cot \left(\theta_{k}\right), \quad s\left(\theta_{k}\right)=r \theta_{k}\left(j+\cot \left(\theta_{k}\right)\right), \quad$ and positive integer $Z$ can be chosen to get the desired accuracy.

\section{AdAPTIVE Modulation}

\subsection{ADR M-QAM/PSK Schemes}

Since the relative improvement with variable-rate variable power adaptive modulation over constant-transmit-power-variable-rate adaptive modulation scheme in fading channels is only marginal [26], in this article we will consider only the latter owing to its reduced implementation complexity. In the context of CAF relay network, the destination node only needs to compute and convey the information on the total (effective) received SNR to the source node for it to select an appropriate transmission rate while keeping the total transmit power $P_{s, d}+\sum_{i=1}^{N} P_{i, d}=P_{T}$ constant. However, in order to determine the optimal power assignment among all the nodes in cooperation, the source node also requires the knowledge of channel gains between the cooperating relays and the destination node.

Generally, it has been observed that a single exponential form cannot give accurate representation of erfc function, and we also evaluated that, BER expression with two exponential terms approximate well the exact (between the range $10^{-1}$ and $10^{-7}$ ). Moreover, evident from [27, eq. (5.2)], the BER expression uses two exponential term, approximate well the exact BERs. This approximate expression simplifies the adaptive modulation design, and facilitates the performance analysis in cooperative relay network under generalized fading environments. So, that in this article we have considered the BER expression with two exponential term.

The BER for M-QAM/PSK modulation, over an additive white Gaussian noise (AWGN) channel is given by [27]

$$
P_{e} \approx a_{n} \exp \left(-b_{n} \gamma\right)+c_{n} \exp \left(-2 b_{n} \gamma\right)
$$

where, $P_{e}$ is the target BER for a given channel $\operatorname{SNR}(\gamma)$ and $n$ is the mode index. Parameters $a_{n}, b_{n}$, and $c_{n}$ in (9) depend on the selection of a particular mode, and are obtained from [27, Table5.1].

In ADR M-QAM/PSK system, the range of the effective received SNR is divided into T+1 fading regions. When the fading causes the total received SNR to fall into the $n$-th region $(n=0,1, \ldots$, T), the constellation size $M_{n}$ is employed for transmission. Also, the SNR thresholds for partitioning of the total received SNR depends on the target BER level, $P_{e}$. The region boundary $\gamma_{n}$ is chosen for the corresponding transmission mode $n$ to be the minimum SNR required to achieve $P_{e}$, which can be easily shown by inverting (9) and is given by [27]: 


$$
\begin{aligned}
& \gamma_{n} \approx-\frac{1}{b_{n}} \ln \left(\frac{-a_{n}+\sqrt{a_{n}^{2}+4 c_{n} P_{e}}}{2 c_{n}}\right), n=1,2,3 \ldots \ldots ., T \\
& \gamma_{T+1}=+\infty .
\end{aligned}
$$

\subsection{Outage Probability}

When the total received SNR falls below the region boundary threshold $\gamma_{1}$ (i.e., $\gamma_{1}$ is obtained by substituting $a_{1}, b_{1}$ and $c_{1}$ from Table $\mathrm{I}$ in (10)), the source node $\mathrm{S}$ ceases transmission because the prescribed target BER cannot be satisfied even with the smallest constellation size. The probability of such an outage event is given by $P_{\text {out }}=F_{\gamma}\left(\gamma_{1}\right)$, where the CDF term can be evaluated efficiently using (8) given that the MGF of $\gamma$ is available or alternatively, by setting $s=0$ in our derived closed form marginal MGF expression in (6). Although note that we have derived a closed-form expression of the marginal MGF in (6) for the case of i.n.d Nakagami-m fading (positive integer $m$ ) from (5), it is very simpler to program and evaluate the desired outage probability using (8) (in conjunction with (5)). The latter approach circumvents the need to determine the coefficients of partial fractions as in (6) (or evaluate the corresponding higher order derivatives of a product term) which becomes cumbersome especially when a large number of cooperative relays are being considered.

\subsection{Average Normalized Spectral Efficiency}

The normalized mean achievable spectral efficiency for ADR M-QAM/PSK is given by the weighted sum of the data rates in each of the partitioned regions [11], viz.,

$$
\frac{R_{a d r}}{B}=\frac{1}{N+1} \sum_{n=1}^{T} n p_{n}
$$

where $p_{n}$ denotes the transmission mode selection probability (i.e., probability that the total received SNR falls in the $n$-th partition region):

$$
p_{n}=\int_{\gamma_{n}}^{\gamma_{n+1}} f_{\gamma}(\gamma) d \gamma=F_{\gamma}\left(\gamma_{n+1}\right)-F_{\gamma}\left(\gamma_{n}\right)
$$

\subsection{Average Bit Error Rate of ADR M-QAM/M-PSK}

The ABER of ADR M-QAM/PSK can be calculated as the ratio of average number of error bits per transmission divided by the average number of bits per transmission [26], viz.,

$$
A B E R_{a d r}=\frac{\sum_{n=1}^{T} n \overline{B E R_{n}}}{\sum_{n=1}^{T} n\left[F_{\gamma}\left(\gamma_{n+1}\right)-F_{\gamma}\left(\gamma_{n}\right)\right]},
$$

where

$$
\begin{aligned}
\overline{B E R_{n}} & =\int_{\gamma_{n}}^{\gamma_{n+1}}\left\{a_{n} \exp \left(-b_{n} \gamma\right)+c_{n} \exp \left(-2 b_{n} \gamma\right)\right\} f_{\gamma}(\gamma) d \gamma \\
& =a_{n}\left[\phi_{\gamma}\left(b_{n}, \gamma_{n+1}\right)-\phi_{\gamma}\left(b_{n}, \gamma_{n}\right)\right]+c_{n}\left[\phi_{\gamma}\left(2 b_{n}, \gamma_{n+1}\right)-\phi_{\gamma}\left(2 b_{n}, \gamma_{n}\right)\right]
\end{aligned}
$$


and the term $\phi_{\gamma}(\beta, \alpha)=\int_{\alpha}^{\infty} e^{-\beta \gamma} f_{\gamma}(\gamma) d \gamma$ in (14) denotes the marginal MGF of total received SNR. Note that as we explained in section 3.2, the closed-form formula for $\phi_{\gamma}(\beta, \alpha)$ in (14) is readily available in (6) for single relay i.n.d Rayleigh and Nakagami fading channel. However, the closed form marginal MGF for higher number of relays remains intractable. Even for the case of single 2-hop CAF relay system with the Rice fading channel, solution is not available. Therefore, in this article, we creatively circumvent this problem by expressing the marginal MGF of any fading statistics as a difference of two "CDF" terms of an auxiliary function in conjunction with (8) as detailed in [12, Appendix C], viz.,

$$
\overline{B E R_{n}}=a_{n}\left[F_{\hat{\gamma}_{a}}\left(\gamma_{n+1}\right)-F_{\hat{\gamma}_{a}}\left(\gamma_{n}\right)\right]+c_{n}\left[F_{\hat{\gamma}_{b}}\left(\gamma_{n+1}\right)-F_{\hat{\gamma}_{b}}\left(\gamma_{n}\right)\right]
$$

where $F_{\hat{\gamma}_{a}}(x)$ and $F_{\hat{\gamma}_{b}}(x)$ in (15) can be evaluated efficiently via (8), but using the "MGF" formulas of auxiliary functions (i.e., $\phi_{\gamma_{a}}(s)=\phi_{\gamma}\left(s+b_{n}\right)$ and $\left.\phi_{\gamma_{b}}(s)=\phi_{\gamma}\left(s+2 b_{n}\right)\right)$. Moreover, it is important to note that the above approach is generalized to any fading environment and not restricted to specific number of cooperative relays, as long as the overall MGF of the fading environment is readily available. (For, example here we presented results for the Rayleigh, Rice and the Nakagami-m fading channels, computed using our generalized analytical framework).

\section{NUMERICAL RESUltS}

In this section, selected numerical results are provided for the outage probability, normalized mean achievable spectral efficiency, and ABER performance metrics of CAF relay networks with adaptive M-QAM/M-PSK transmission in generalized fading environments. The following mean link SNRs (arbitrarily chosen) will be used to generate the plots, unless stated otherwise: $\Omega_{s, 1}=$ $E_{s} / N_{0}, \Omega_{s, 2}=0.5 E_{s} / N_{0}, \Omega_{1, d}=0.5 E_{s} / N_{0}, \Omega_{2, d}=E_{s} / N_{0}$, and $\Omega_{s, d}=0.2 E_{s} / N_{0}$. We have considered following three cases, for the values of fading severity index on different wireless links, when evaluating the performance of CAF relay networks in fading environment with i.n.d fading statistics. (Note: $m_{a, b}$ denotes the fading severity index of link $a-b$ ):

Case 1: $m_{s, 1}=m_{s, 2}=m_{1, d}=m_{2, d}=m_{s, d}=1 ;$ Case 2: $m_{s, 1}=4, m_{s, 2}=4, m_{1, d}=2, m_{2, d}=2, m_{s, d}=1$; Case 3: $m_{s, 1}=2, m_{s, 2}=2, m_{1, d}=4, m_{2, d}=4, m_{s, d}=1$.

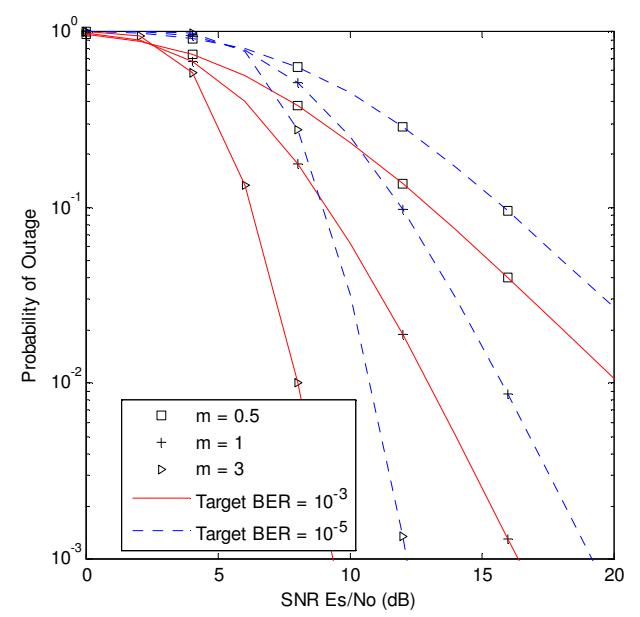

Figure 2: Outage probability of CAF network with two cooperating relays in an i.i.d Nakagami-m fading channel 
Figure 2 depicts the probability of outage for CAF relay network with two cooperating relays in an i.i.d Nakagami-m fading channel evaluated, using [19] $\phi_{n_{n}}^{(H M)}(s)={ }_{2} F_{1}\left(m, 2 m ; m+\frac{1}{2} ;-\frac{\Omega s}{4 m}\right)$ (in conjunction with (8)). Figure 2 also reveals that the outage performance for the curve with $\mathrm{m}=3$ are considerably better than $\mathrm{m}=0.5$ and $\mathrm{m}=1$ (i.e., for the special case of Rayleigh fading), as anticipated. This is because for a fixed set of mean channel SNRs, better link conditions (i.e., higher fading severity index) translate into lower probability of outage. But the asymptotic "diversity gain" (i.e., slope of the curve) appears to be independent of the target BER, as anticipated.

Figure 3 illustrates ABER performance curves for a CAF relay network in an i.n.d Nakagami-m fading environment, with ADR M-PSK at target BER of $10^{-3}$. Figure 3 also reveals that the performance of Case 2 and Case 3 are considerably better than Case 1 (i.e., special case of Rayleigh fading). This is because for a fixed set of mean channel SNRs, better link conditions (i.e., higher fading severity index) translate into lower ABER.

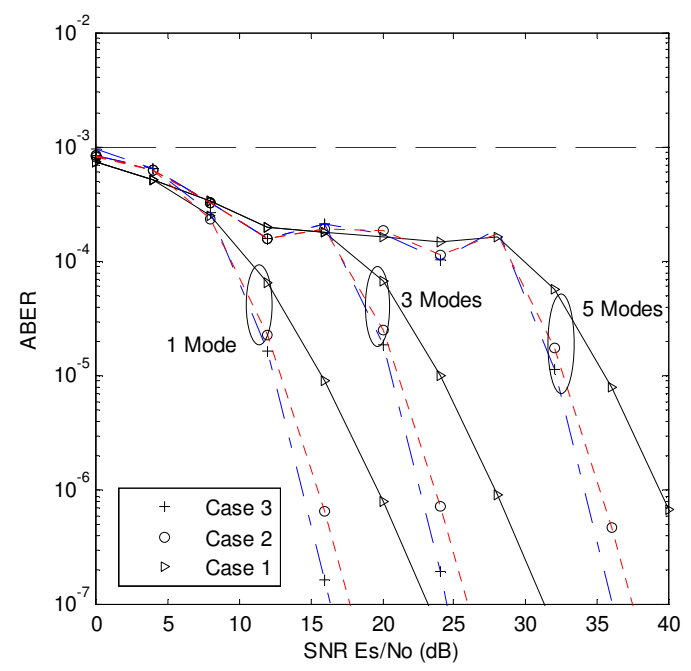

Figure 3: ABER performance curves for adaptive M-PSK in an i.n.d. Nakagami-m fading channel with two cooperative relays ("for $\phi_{\gamma}^{(U B)}$ case")

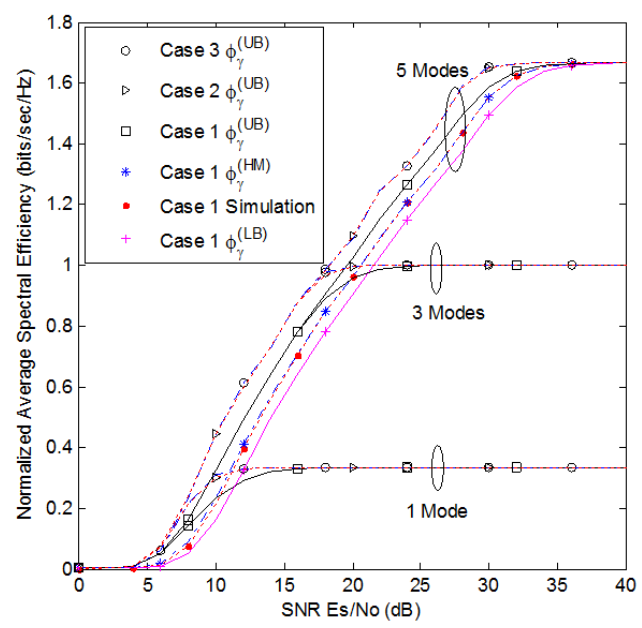

Figure 4: Mean achievable rates for ADR M-PSK in an i.n.d Nakagami-m fading environment with two cooperative relays 
The parameters used to generate Figure 4 are identical to that of Figure 3. It is evident from Figure 4 that by increasing the number of transmission-rate modes in ADR M-PSK directly translates into improved spectral efficiency. However, this improvement is achieved at the expense of increased ABER (see Figure 3). Monte-Carlo results are also given, which shows a good agreement with the curve corresponding to the "Harmonic Mean, (i.e., $\phi_{\gamma}^{(H M)}$ )" case, as anticipated (i.e., generated using [22],

$\phi_{n}^{(H M)}(s)=\left[\left(1 / \Omega_{s, i}-1 / \Omega_{i, d}\right)^{2}+\left(1 / \Omega_{s, i}+1 / \Omega_{i, d}\right) s\right] / \Delta^{2}+\frac{2 s}{\Delta^{3} \Omega_{s, i} \Omega_{i, d}} \ln \left(\left(s+\Delta+\frac{1}{\Omega_{s, i}}+\frac{1}{\Omega_{i, d}}\right)^{2} \frac{\Omega_{s, i} \Omega_{i, d}}{4}\right)$

where $\Omega_{a, b}=E\left[\gamma_{a, b}\right]$ corresponds to the mean link SNR and $\left.\Delta=\sqrt{\left(1 / \Omega_{s, i}-1 / \Omega_{i, d}\right)^{2}+2 s\left(1 / \Omega_{s, i}+1 / \Omega_{i, d}\right)+s^{2}}\right)$.

Although authors in [11] have considered the mean spectral efficiency of CAF relay networks in i.n.d Rayleigh fading environments, but their framework does not lend itself to the analysis of the "Harmonic Mean" case or generalized to other fading channels, where as our generalized analytical framework encapsulates all these cases in a unified way (i.e., see Figure 4. for i.n.d Nakagami-m along with special case (i.e., case 1) for Rayleigh fading and Figure 5 for Rice fading channel).

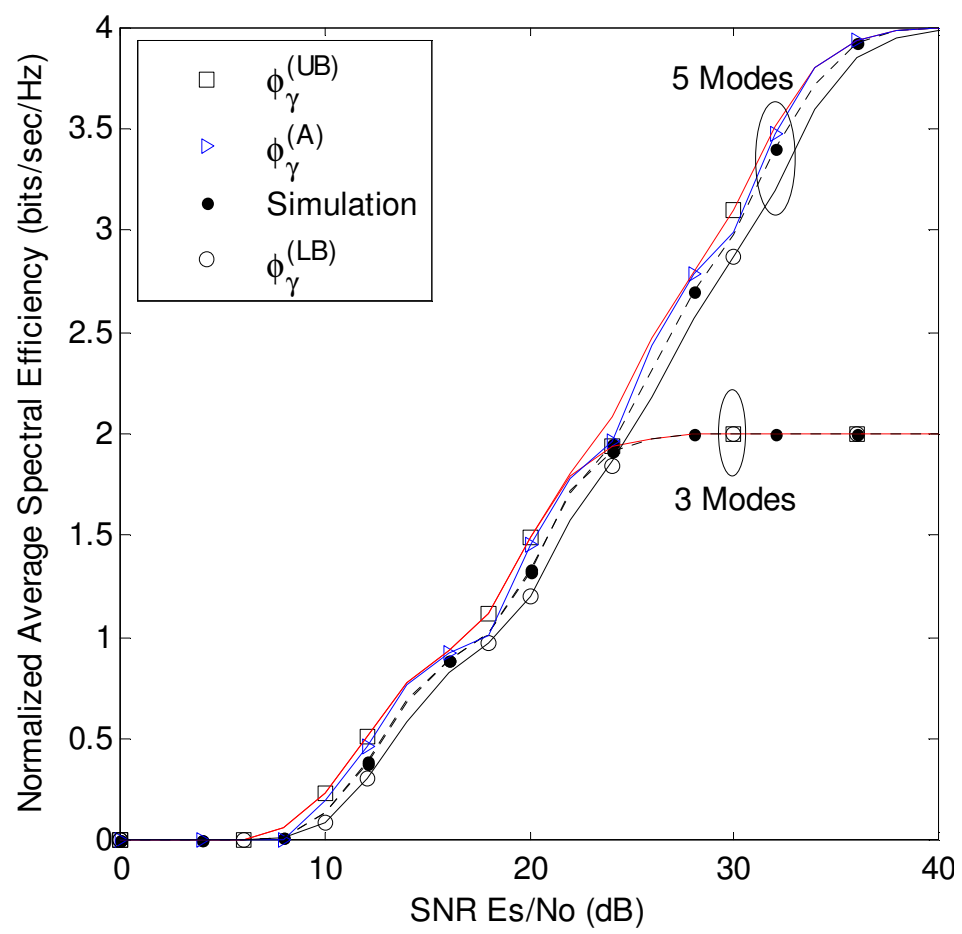

Figure 5: Mean achievable rates for ADR M-QAM in an i.n.d Rice fading environment with one cooperative relay

Figure 5 depicts the mean achievable spectral efficiency curves for a CAF relay network in an i.n.d Rice fading environment with ADR M-QAM at target BER of $10^{-3}$. Curves with $\phi_{\gamma}^{(U B)}$ can be generated using [12]

$\phi_{r_{i}}^{(U B)}(s)=\sum_{\substack{k \in\{(s, i)(i, d)\} \\ j \neq k}} \mathrm{~A}_{k} e^{-K_{k}} 2 I\left[\sqrt{2 A_{j}}, \sqrt{2 K_{j}}, \sqrt{2 A_{k} K_{k}}, 2\left(s+A_{k}\right)\right]$ 
in conjunction with (2), while the MGF for the lower bound may be computed as $\phi_{\gamma_{i}}^{(L B)}(s)=\phi_{\gamma_{i}}^{(U B)}(s / 2)$.

$\operatorname{In}(17), A_{i}=1+K_{i} / \Omega_{i}, I[a, b, c, d]=\frac{1}{d} e^{\frac{c^{2}}{2 d}} Q\left(b \sqrt{\frac{d}{\left(d+a^{2}\right)}}, \frac{a c}{\sqrt{d\left(d+a^{2}\right)}}\right)-\frac{a^{2}}{d\left(d+a^{2}\right)} e^{\frac{c^{2}-b^{2} d}{2\left(d+a^{2}\right)}} I_{0}\left(\frac{a b c}{\left(d+a^{2}\right)}\right)$,

$K_{k}$ is the Rice fading parameter, $I_{0}($.$) is the zero order modified Bessel function and Q(.,$.$) is the$ first order Marcum $Q$-function and can be evaluated using [12, eq., (10)]

$$
Q_{u}(\sqrt{2 a}, \sqrt{b})=1-\sum_{k=0}^{\infty} \frac{a^{k} e^{-a}}{k !} \frac{G\left(u+k, \frac{b}{2}\right)}{\Gamma(u+k)}
$$

where $G(.,$.$) is the lower incomplete gamma function, which is defined by G(a, x)=\int_{0}^{x} t^{a-1} e^{-t} d t$.

As expected, as the fading index increases, the spectral efficiency increases. We also noticed that the curve corresponding to Approximate MGF (i.e., $\phi_{\gamma}^{(A)}$ ) method is very close to the Monte-Carlo simulation. Therefore, introducing this novel technique based on "approximate MGF" allows us to simplify the computation complexity of achievable spectral efficiency; outage probability as well as ABER of CAF relay system in generalized fading environments by simply replacing appropriate single channel MGF as readily available in [25, Table 2.2]. To the best of our knowledge, a similar study that incorporates M-QAM/M-PSK modulation for CAF relay networks in the Rice fading environment has never been undertaken previously in the literature.

The main reason is that, the evaluation of mean spectral efficiency, ABER and outage probability require a tractable expression for the PDF, CDF and/or MGF of end-to-end SNR, which has been difficult to obtain for the Rice fading channel. It is worth to mention that the exact MGF of harmonic mean SNR for the relayed path presented in [13, Eq. (5) and Table 1] for the Rice fading channel is not tractable as it involves the integral of the product of two infinite series (with Bessel function arguments).

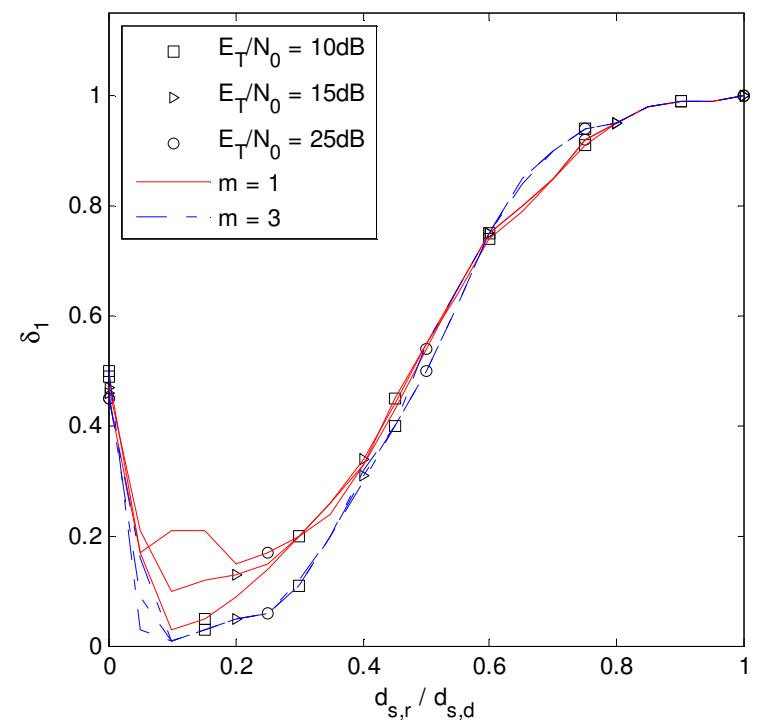

Figure 6: Transmit power assignment, $\delta_{1}$ of a CAF network consisting of 1 relay $(\mathrm{N}=1)$ as a function of position of relay (i.e., ratio of distances, $d_{s, r} / d_{s, d}$ ) in an i.n.d Nakagami-m channels

$$
\text { ("for } \phi_{\gamma}^{(U B)} \text { case") }
$$




\section{Node Placement based Optical Power Allocation}

We have assumed following mean link SNRs between different links (source-relay, relaydestination and source-destination) to generate Figures 6-8 (which take into account the relative distances between the cooperating nodes and for each node $\Omega_{q}$ is given by: $\Omega_{q}=\left(d_{q} / d_{s, d}\right)^{-L_{p}} c \delta_{q} E_{r} / N_{o}$ where $q \in\{(s, d),(s, i),(i, d)\}, c$ is a constant that is related to the carrier wavelength (i.e., $c=(\lambda / 4 \pi)^{2}$ for free space path loss $), L_{p}$ is the path loss exponent, and $d_{a, b}$ denotes the distance between links $a-b$. For $N=1, \delta_{s, d}=\delta_{s, i}=\delta_{1}=P_{s, i} / P_{T}$ and $\delta_{1, d}=\delta_{2}=1-\delta_{1}$. For $N=2$, $\delta_{2, d}=\delta_{3}=1-\left(\delta_{1}+\delta_{2}\right)$. Values $c=10^{-1}$ and $L_{p}=4$ have been chosen to generate the plots.

Figure 6 shows the transmit power assignment, $\delta_{1}$ at an optimum value of spectral efficiency, as a function of distance ratio (i.e., relay position with respect to source and destination) of a cooperative relay network consisting of 1 relay $(\mathrm{N}=1)$ using M-QAM 5 modes under the Nakagami-m fading channels. From the Figure we notice that the power assignment during different links strongly depends on the position of the relays. Additionally, with the plot corresponding to $\mathrm{m}=1$ and $\mathrm{m}=3$, we observe that; the effect of fade distribution is very small on power allocation, compared to the node placement.

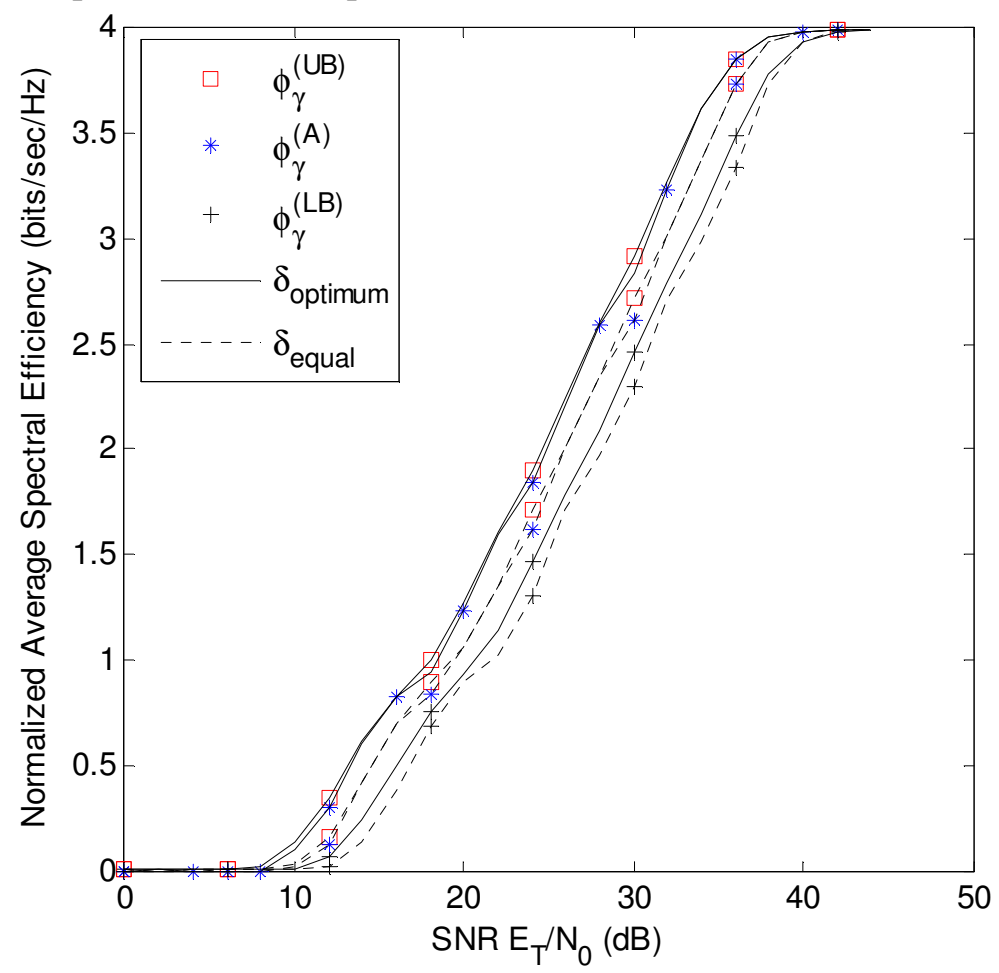

Figure 7: Comparison between average spectral efficiencies of CAF relay network with the optimal and the equal power allocation strategies in an i.n.d Nakagami-m fading channels

Figure 7 shows the comparison between average spectral efficiencies of the optimal and the equal power allocation strategies with one relay diversity in i.n.d Nakagami-m fading environment using 5 modes adaptive M-QAM. To generate Figure 7, we have arbitrarily chosen normalized distances with respect to $d_{s, d}: d_{s, 1}=0.3$ and $d_{1, d}=0.7$. Moreover, the fading severity index for the wireless links are assumed to be $m_{s, d}=3, m_{s, 1}=3$ and $m_{1, d}=3$. As shown in Figure 6, for this relay position the optimum power allocation factor are $\left(\delta_{1}=0.11, \delta_{2}=0.89\right)$. From Figure 7, we noticed that, the spectral efficiency with approximate MGF $\phi_{\gamma}^{(A)}$ is in good agreement with the curve 
corresponding to $\phi_{\gamma}^{(U B)}$ case. Moreover, we observe that the mean spectral efficiency of ADR with optimal power assignment among collaborating nodes is comparatively higher than that of the equal power allocation strategy. However, it should be recognized that this improvement is attained with complete CSI (i.e., the knowledge of channel gain of all links) compared to the limited CSI (i.e., only the knowledge of the total received SNR is needed at the source node) with the latter scheme.

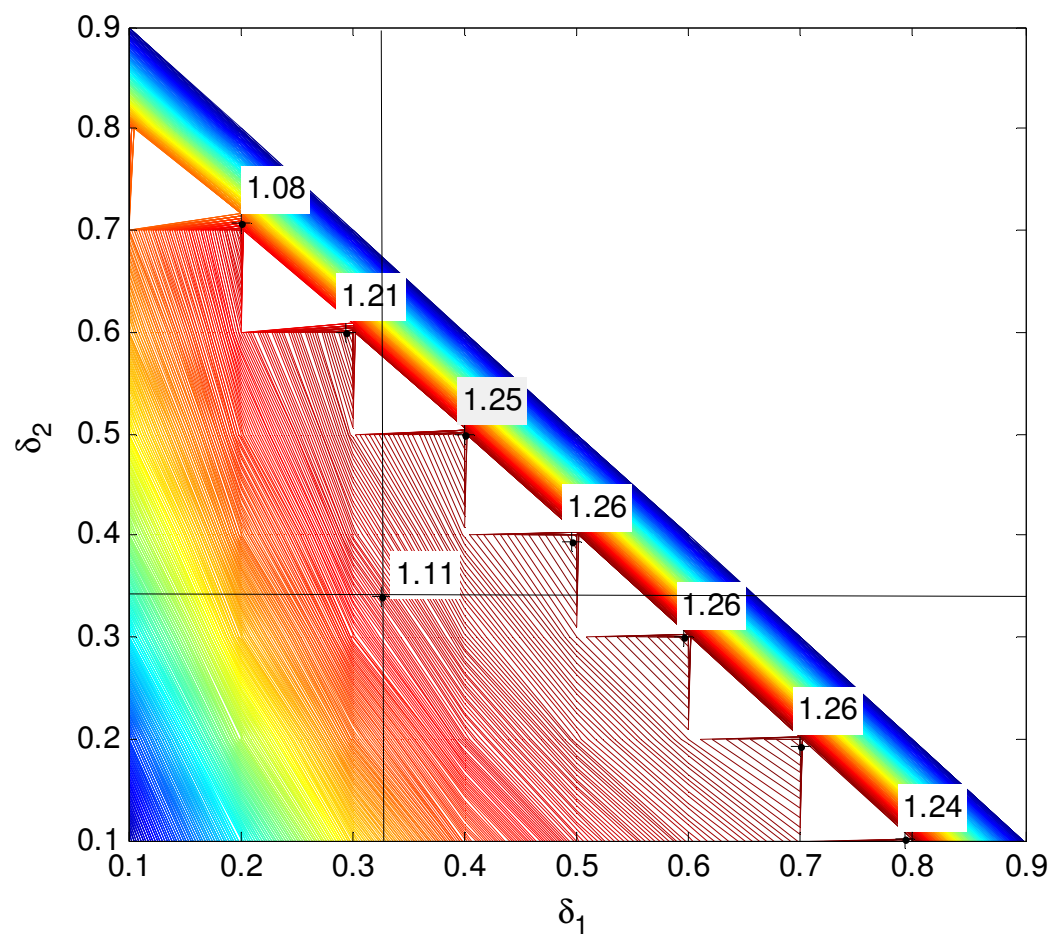

Figure 8: Contour plot for average spectral efficiency (using $\phi_{\gamma}^{(A)}(\cdot)$ ) as a function of $\delta_{1}$ and $\delta_{2}$ consisting of two relays $(\mathrm{N}=2)$ in the Rice fading channel with $\mathrm{K}=3$.

In Figure 8 the contour plot is used to determine the optimum values of $\delta_{1}$ and $\delta_{2}$ for $N=2$, that maximizes the average spectral efficiency at $E_{T} / N_{o}=25 \mathrm{~dB}$ using 3 modes adaptive M-QAM. The optimum values are normally found in the least dense lines/curves. To generate Figure 8 , we have arbitrarily chosen normalized distances $d_{s, 1}=0.2, d_{1, d}=0.8, d_{s, 2}=0.7, d_{2, d}=0.3$ and the Rice fading parameters $\mathrm{K}=3$ on each links. We observe that the multiple optimum values of $\delta_{1}$ and $\delta_{2}$ respective pair, $\{(0.5,0.4),(0.6,0.3),(0.7,0.2)\}$ give the same spectral efficiency of 1.26 .

Comparatively, the spectral efficiency with the equal power allocation (i.e., $\delta_{1}=\delta_{2}=1 / 3$ shown by solid line across the axis) is 1.11 , which is lower compared to value, 1.26 for the optimum power allocation. This shows that the choice of the relay location is very important in optimum power allocation. To the best of our knowledge analysis on relay position based optimal power allocation with Rice fading channel has not been presented in earlier publications. 


\section{Conclusions}

In this paper, we analyzed the performance of Channel Side Information assisted Cooperative Amplified and Forward (CAF) relay networks that employ both the node placement based optimal power allocation strategy among collaborating nodes and adaptive M-QAM/M-PSK technique in the generalized wireless fading environments. In particular, we advocate a simple yet unified numerical approach based on the marginal MGF of the total received SNR and derived analytical expressions for the ABER, mean achievable spectral efficiency, and outage probability performance metrics. The significant advantage of this proposed framework is, it sufficiently general to characterize the performance of adaptive-link CAF relay networks over a wide range of fading distributions (i.e., it is not only restricted to Rayleigh fading or i.i.d Nakagami fading but also support rice as well as mixed fading channels) with independent but non-identically distributed (i.n.d) fading statistics across the spatially distributed diversity paths. Moreover, to prove the generality/flexibility of this proposed work, we applied this flexible mathematical framework to present the spectral efficiency analysis of Rice fading channel along with optimal power allocation strategy in a simpler way. Additionally, in an attempt to further simplify the computation complexity, we also employ the use of an "approximate MGF expression" to compute the system performance metrics over the generalized fading channel by utilizing our mathematical framework. Employing the above novel approach based on "approximate MGF" allows us to simplify the computation complexity of achievable spectral efficiency as well as ABER of CAF relay system in the generalized fading environments by simply replacing appropriate single channel MGF as readily available. Numerical results reveal that the optimal transmit power allocation among cooperative nodes in a practical CAF relay topologies could lead to a further substantial increase in the mean spectral efficiency compared to the equal power assignment case but at the expense of higher network overhead. In summary, our derived analytical framework is very flexible to incorporate wide range of fading channels. Also, it is compatible with MGF of upper/lower bounds, Approximate MGF and tight bound over wide range of fading channels and can be used to derive analysis of mixed fading channel as well.

More importantly it can be easily incorporated to support the analysis for optimal power allocation and relay placement based power allocation strategies.

\section{APPENDiX A}

In this appendix, we will derive the decomposition of closed form MGF for upper bound of $\gamma$ in (2). For instance the MGF for upper bound of $\gamma_{i}^{(U B)}$ for two-hops relayed path with i.n.d statistics can be expressed as [12]

$$
\phi_{\gamma_{i}}^{(U B)}(s)=\sum_{\substack{k \in\{(s, i),(i, d)\} \\ j \neq k}} \frac{\Gamma\left(m_{k}+m_{j}\right)}{m_{k} \Gamma\left(m_{k}\right) \Gamma\left(m_{j}\right)}\left(\frac{\Omega_{j} m_{k}}{s \Omega_{j} \Omega_{k}+\Omega_{j} m_{k}+\Omega_{k} m_{j}}\right)^{m_{k}}{ }_{2} F_{1}\left(1-m_{j}, m_{k} ; 1+m_{k} ; \frac{\left(s \Omega_{k}+m_{k}\right) \Omega_{j}}{s \Omega_{j} \Omega_{k}+\Omega_{j} m_{k}+\Omega_{k} m_{j}}\right)
$$

where ${ }_{2} F_{1}(a, b ; \quad c ; \quad z)$ is gauss hypergeometric function. Using the identity ${ }_{2} F_{1}(-U, b ; c ; V)=\sum_{u=0}^{U}(-1)^{-u}\left(\begin{array}{l}U \\ u\end{array}\right) \frac{(b)_{u}}{(c)_{u}} V^{u} ;$ where $\mathrm{U}$ is integer and ${ }_{(b)_{u}}=\frac{\Gamma(a+u)}{\Gamma(a)}$ is pochhammer symbol,

(A.1) can be rewritten for special case of integer $m$ as 


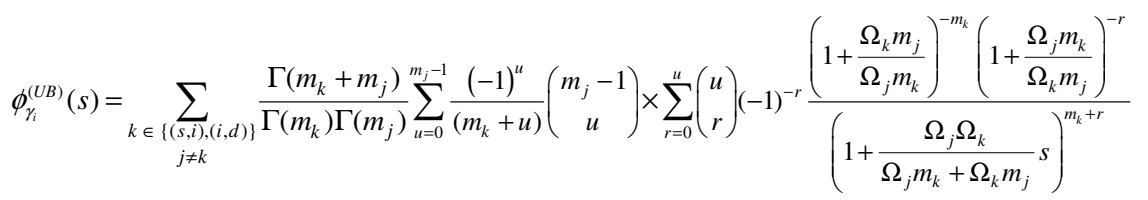

Multiplying MGF of SNR of the source to destination link $\left(i . e ., \phi_{\gamma_{s . d}}(s)=\left(1+\frac{\Omega_{s}}{m_{s}} s\right)^{-m_{s}}\right)$ with $\phi_{\gamma_{i}}^{(U B)}(s)$ in (A.2), we can write MGF for upper bound of $\gamma$ as,

$\phi_{\gamma}^{(U B)}(s)=\sum_{\substack{k \in\{(s, i),(i, d)\} \\ j \neq k}} \frac{\Gamma\left(m_{k}+m_{j}\right)}{\Gamma\left(m_{k}\right) \Gamma\left(m_{j}\right)} \sum_{u=0}^{m_{j}-1} \frac{(-1)^{u}}{\left(m_{k}+u\right)}\left(\begin{array}{c}m_{j}-1 \\ u\end{array}\right) \times \sum_{r=0}^{u}\left(\begin{array}{l}u \\ r\end{array}\right)(-1)^{-r} \frac{\left(1+\frac{\Omega_{k} m_{j}}{\Omega_{j} m_{k}}\right)^{-m_{k}}\left(1+\frac{\Omega_{j} m_{k}}{\Omega_{k} m_{j}}\right)^{-r}}{\left(1+\frac{\Omega_{s}}{m_{s}} s\right)^{m_{s}}\left(1+\frac{\Omega_{j} \Omega_{k}}{\Omega_{j} m_{k}+\Omega_{k} m_{j}} s\right)^{m_{k}+r}}$

Using partial fractions (A.3) can be rewritten as,

$$
\begin{aligned}
& \phi_{\gamma}^{(U B)}(s)=\sum_{\substack{k \in\{(s, i)(i, d)\} \\
j \neq k}} \frac{\Gamma\left(m_{k}+m_{j}\right)}{\Gamma\left(m_{k}\right) \Gamma\left(m_{j}\right)} \sum_{u=0}^{m_{j}-1} \frac{(-1)^{u}}{\left(m_{k}+u\right)}\left(\begin{array}{c}
m_{j}-1 \\
u
\end{array}\right) \times \sum_{r=0}^{u}\left(\begin{array}{l}
u \\
r
\end{array}\right)(-1)^{-r}\left(1+\frac{\Omega_{k} m_{j}}{\Omega_{j} m_{k}}\right)^{-m_{k}}\left(1+\frac{\Omega_{j} m_{k}}{\Omega_{k} m_{j}}\right)^{-r} \\
& \times\left[\sum_{p=1}^{m_{s}} \frac{\alpha_{p}}{\left(1+\frac{\Omega_{s}}{m_{s}} s\right)^{p}}+\sum_{q=1}^{m_{k}+r} \frac{\alpha_{q}}{\left(1+\frac{\Omega_{j} \Omega_{k}}{\Omega_{j} m_{k}+\Omega_{k} m_{j}} s\right)^{q}}\right]
\end{aligned}
$$

where

$$
\alpha_{p}=\frac{\left(\frac{m_{s}}{\Omega_{s}}\right)^{m_{s}-p}(-1)^{m_{s}-p}\left(\frac{\Omega_{j} \Omega_{k}}{\Omega_{j} m_{k}+\Omega_{k} m_{j}}\right)^{m_{s}-p}}{\left(1-\frac{\Omega_{j} \Omega_{k}}{\Omega_{j} m_{k}+\Omega_{k} m_{j}}\left(\frac{m_{s}}{\Omega_{s}}\right)\right)^{m_{s}-p+1}} \text { and } \alpha_{q}=\frac{\left(\frac{\Omega_{j} m_{k}+\Omega_{k} m_{j}}{\Omega_{j} \Omega_{k}}\right)^{m_{k}+r-q}(-1)^{m_{k}+r-q}\left(\frac{\Omega_{s}}{m_{s}}\right)^{m_{k}+r-q}}{\left(1-\frac{\Omega_{s}}{m_{s}}\left(\frac{\Omega_{j} m_{k}+\Omega_{k} m_{j}}{\Omega_{j} \Omega_{k}}\right)\right)^{m_{k}+r-q+1}}
$$

\section{APPENDIX B}

In order to derive the corresponding marginal MGF of the MGF expression in (A.4), we define a generic MGF function as

$$
F(a, k, s)=(1+a s)^{-k}
$$

where $s$ is the Laplace variable.

Taking the inverse Laplace transform of (B.1) we can easily obtain the PDF given by

$$
f(a, k, \gamma)=a^{-i} \frac{\gamma^{k-1}}{\Gamma(k)} e^{-\frac{\gamma}{a}}
$$

Therefore the marginal MGF expression is given by 


$$
M\left(a, k, s, \gamma_{0}\right)=\frac{a^{-k}}{\Gamma(k)} \int_{0}^{\gamma_{0}} \gamma^{k-1} e^{-\left(s+\frac{1}{a}\right) \gamma} d \gamma
$$

Utilizing identity in [28, eq. (3.351.1.8)], (B.3) can be written as,

$$
\begin{aligned}
M\left(a, k, s, \gamma_{0}\right) & =\frac{a^{-k}}{\Gamma(k)}\left(s+\frac{1}{a}\right)^{-k} G\left(k,\left(s+\frac{1}{a}\right) \gamma_{0}\right) \\
& =\frac{G\left(k,\left(s+\frac{1}{a}\right) \gamma_{0}\right)}{\Gamma(k)(1+a s)^{k}}
\end{aligned}
$$

where $G(v, x)=\int_{o}^{x} t^{p-1} e^{-t} d t$ is lower incomplete gamma function.

\section{ACKNOWLEDGEMENT}

This work is supported in part by funding from the US Army Research Office, US Air Force Research Laboratory/Clarkson Aerospace, and the National Science Foundation.

\section{REFERENCES}

[1] A. Madsen and J. Zhang, (2005) "Capacity Bounds and Power Allocation for Wireless Relay Channels," IEEE Trans. Info. Theory, vol. 51, pp. 2020-2040.

[2] Y. Zhao, R. Adve, and T. Lim, (2007) "Improving Amplify-and-Forward Relay Networks: Optimal Power Allocation versus Selection,” IEEE Trans. Wireless Comm., pp. 3114-3123.

[3] M. Hasna, (2005) "On the Capacity of Cooperative Diversity Systems with Adaptive Modulation," Proc. International Conference on Wireless and Optical Communication Networks, pp. 432-436.

[4] S. Ikki, M. Uysal, and M. H. Ahmed, (2009) "Joint Optimization of Power Allocation and Relay Location for Decode- and-Forward Dual-Hop Systems over Nakagami-m Fading Channels," IEEE GLOBECOMM Conf., pp. 1-6.

[5] R. A. Renani, R. Saadat, M. R. Aref and G. Mirjalily, (2011) "Power Allocation for IncrementalSelective Decode- and-Forward Cooperative Communication over Rician Fading Channels," IEEE International conference on Advanced Communication Technology, pp. 730-734.

[6] Mehrdad Taki, Mohammad Sadeghi, (2014) "Joint Relay Selection and Adaptation of Modulation Coding and Transmit Power for Spectral Efficiency Optimisation in Amplify-Forward Relay Network", IET Communications, vol. 8, pp. 1955-1964.

[7] Q. Zhao, H. Li. and Pu Wang, (2008) "Performance of Cooperative Relay with Binary Modulation in Nakagami-m Fading Channels," IEEE Trans. Vehicular Tech., vol. 57, no. 5, pp. 3310-3315.

[8] T. Nechiporenko, P. Kalansuriya, and C. Tellambura, (2009) "Performance of Optimum Switching Adaptive M-QAM for Amplify-and-Forward Relays,” IEEE Trans. Vehic. Tech., vol. 58, pp. 22582268.

[9] K. Hwang, Y. Ko, and M. Alouini, (2008) "Performance Analysis of Opportunistic Incremental Relaying with Adaptive Modulation over Cooperative Networks," Proc. IEEE Int. Symp. Wireless Pervasive Compu., May 2008, pp. 586-590.

[10] P. Kalansuriya, and C. Tellambura, (2009) "Performance Analysis of Decode-and-Forward Relay Network Cooperative Network under Adaptive M-QAM," Proc. IEEE International Conference on Communications, pp. 298-303.

[11] T. Nechiporenko, K. Phan, C. Tellambura and H. Nguyen, (2008) "Performance Analysis of Adaptive M-QAM for Rayleigh Fading Cooperative Systems," Proc. IEEE International Conference on Communications, pp. 3393-3399. 
[12] Bhuvan Modi, A. Annamalai, O. Olabiyi, and R. Chembil Palat, (2012) "Ergodic Capacity Analyses of Cooperative Amplify and Forward Relay Networks over Rice and Nakagami Fading Channels," International Journal of Wireless and Mobile Networks, vol. 4, no. 1, pp. 97-116.

[13] M. Di Renzo, F. Graziosi and F. Santucci, (2009) "A Unified Framework for Performance Analysis of CSI-Assisted Cooperative Communications over Fading Channels," IEEE Trans. on Communications, vol. 57, pp. 2551-2557.

[14] T. Nechiporenko, K. Phan, C. Tellambura and H. Nguyen, (2009) "Capacity of Rayleigh Fading Cooperative Systems under Adaptive Transmission," IEEE Trans. Wireless Comm., vol. 8, pp. 16261631.

[15] R. Chembil Palat, A. Annamalai and J. Reed, (2008) “An Efficient Method for Evaluating Information Outage Probability and Ergodic Capacity of OSTBC Systems,” IEEE Comm. Letters, pp. 191-193.

[16] O. Olabiyi, and A. Annamalai, (2011) "ASER Analysis of Cooperative Non-Regenerative Relay Systems over Generalized Fading Channels," Proc. IEEE International Conference on Computer Communication and Networking, pp. 1-6.

[17] N. Laneman, D. Tse, and G. Wornell, (2004) "Cooperative Diversity in Wireless Networks: Efficient Protocols and Outage Behaviour," IEEE Trans. Information Theory, vol. 50, pp. 3062-3080.

[18] Bhuvan Modi, O. Olabiyi, A. Annamalai and D. Vaman, (2011) "Improving the Spectral Efficiency of Adaptive Modulation in Amplify-and-Forward Cooperative Relay Networks with an Adaptive ARQ Protocol," Proc. IEEE GLOBECOM Conf., pp.1-5.

[19] M. Hasna and M. Alouini, (2004) "Harmonic Mean and End-to-End Performance of Transmission System with Relays," IEEE Trans. Communications, vol. 52, no.1, pp. 130-135.

[20] R. H. Y. Louie, Y. Li, and B. Vucetic, (2008) "Performance Analysis of Beamforming in two Hop Amplify and Forward Relay Networks," in Proc. IEEE International Conference on Communications, May 19-23, pp. 4311-4315.

[21] D.Senarante and C. Tellambura, (2010) "Unified Exact Performance Analysis of Two-Hop Amplifyand-Forward Relaying in Nakagami fading,"IEEE Trans. Veh. Tech., vol. 59, pp. 1529-1534.

[22] W. Su. K. S. Ahmed, and R. Liu K. J., (2008) "Cooperative Communication Protocols in Wireless Networks: Performance Analysis and Optimum Power Allocation," Springer link Wireless Personal Communication, vol. 44 pp. 181-217.

[23] A. Annamalai, B. Modi, and R. Palat, (2011) "On the Ergodic Capacity of Cooperative Analog Relaying with Source Adaptive Transmission Policies," Proc. IEEE Consumer Commu. and Networking Conf., pp. $812-813$.

[24] A. Annamalai, B. Modi, R. Palat, J. Matyjas, (2010) "Tight-bounds on the Ergodic Capacity of Cooperative Analog Relaying with Adaptive Source Transmission Techniques". IEEE International Symposium on Personal, Indoor, and Mobile Radio Communications, pp. 18-23.

[25] M.K. Simon and M-S Alouini, (2005) Digital Communication over Fading Channels, New York: Wiley, 2 edition.

[26] A. Goldsmith and S. Chua, (1997) "Variable-Rate Variable-Power M-QAM for Fading Channels," IEEE Trans. Commun., vol. 45, pp. 1218-1230.

[27] J.D. Gaeddert, (2005) "Parametric Estimation of Stochastic Fading Channels and Their Role in Adaptive Radios," Master Thesis, Virginia polytechnic Institute and State University, USA.

[28] I. S. Gradshteyn and I. M. Ryzhik, (1995) Table of Integrals, Series and Products, Academic Press.

[29] Bhuvan Modi, A. Annamalai, O. Olabiyi, R. Palat (2013) "Ergodic Capacity Analysis of Cooperative Amplify-and-Forward Relay Networks over Generalized Fading Channels". Wiley Journal of Wireless Communications and Mobile Computing, vol. 15, pp. 1259-1273.

\section{AUTHORS}

Dr. Bhuvan Modi received PhD. degree from Prairie View A \& M University, Texas A \& M University System, in 2012. He earned his M.S. degree in Electrical Engineering from Lamar University, United States of America, M.S. degree in Electronics and Communication Engineering from Dharmsinh Desai University, India, and the B.S. degree in Electronics and Communication Engineering from North Gujarat University, India, in 2009, 2002 and 2001, respectively. He is currently working as a Senior Member of Technical Staff at AT\&T Mobility Lab Seattle, WA. Currently Dr. Modi serves as an editorial committee//International

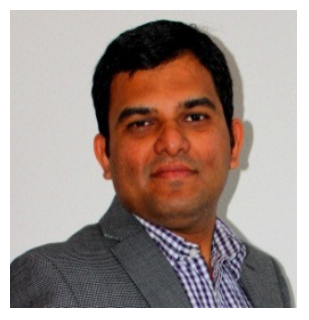
editorial board member for journals and organizations, namely the International Journal of Wireless and 
Mobile Networks (IJWMN), The Standard International Journals (SIJ), the International Journal of Wireless and Mobile Communication for Industrial Systems, Science \& Engineering Research Support Society, and First International Workshop on Wireless and Mobile Communication for Industrial Systems (WMCIS 2015) and has also been invited to serve on the international editorial board committee member for the Journal of Advanced Research in Wireless, Mobile \& Telecommunication. He received 'Student Travel Grant Award' to present his work at the IEEE MILCOM'11 and achieved excellent work appreciation certificate award from Vice President and CEO, AT\&T Mobility Lab for individual contribution towards successfully launch of WiFi Calling Service in the US Market. Over the last few years, Dr. Modi has published over a dozen peer reviewed conference and journal articles. His current research interests include cross-layer design/optimization for adaptive-link cooperative relay networks, 4G/5G Wireless Technologies, Openstack and software-defined radios.

Dr. Annamalai is presently the Director of Center of Excellence for Communication Systems Technology Research, a Texas A\&M Board of Regents approved University Research Center at the Prairie View A\&M University, and a tenured faculty member in the Department of Electrical and Computer Engineering. He has over 20 years of research/teaching experience in wireless communications at Motorola, University of Victoria, Air Force Research Laboratory, Virginia Tech and PVAMU with approximately 200 peer-reviewed publications and 5 book chapters. Dr. Annamalai has been honored by his

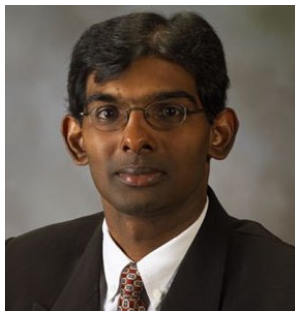
colleagues on numerous occasions for his excellence in research including winning the 2011 Roy G. Perry College of Engineering Outstanding Faculty (Research) Award, IEEE Leon Kirchmayer Prize Paper award, ASEE/AFOSR Summer Faculty Fellowships, NSERC Doctoral Prize, CAGS/UMI Distinguished Doctoral Dissertation Award, IEEE VTS/Motorola Daniel E. Noble Fellowship, among others. He had served on the Editorial Boards of four IEEE journals/transactions in the last 15 years, and has helped to organize a few major IEEE conferences on wireless communications including serving in the capacity of Technical Program Chair of the 2002 IEEE Vehicular Technology Conference in Vancouver, Canada. His current research interests include cooperative spectrum sensing, compressive sensing, cross-layer design for scalable multimedia transmission and cooperative wireless communications.

Dr. Oluwatobi O. Olabiyi received the B.Sc. degree in Electronic and Electrical Engineering from Obafemi Awolowo University, Ile-Ife and M.S. and PhD degrees in Electrical Engineering from Prairie View A\&M University, Texas. Over the last three years, he has co-authored approximately two-dozen peerreviewed conference and journal articles. He was the recipient of the Roy G. Perry College of Engineering Outstanding Masters Student of the Year Award (2011) and the National Society of Black Engineer's Golden Torch Award for Graduate Student of Year (2012). His research interests include dynamic spectrum access, MIMO, cooperative communications, statistical signal processing, compressive sensing, machine-learning and optimization techniques.

Dr. Olusegun O. Odejide received the B.S. degree in Electrical and Electronic engineering from University of Ibadan, Ibadan, Nigeria in 1997. He received the M.S. degree in Computer and Information Systems Engineering from Tennessee State University in 2004 and $\mathrm{PhD}$ degree in Electrical Engineering from Prairie View A\&M University, Prairie View, Texas in 2009. Dr. Odejide has published over a dozen peer reviewed conference and journal articles. His research interests are in the areas of Signal/Image/Video Processing and Communication Systems.
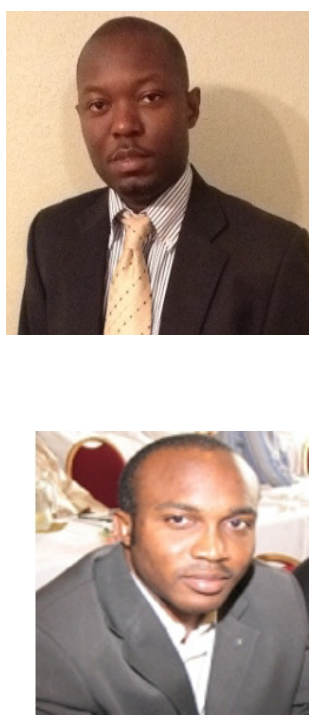